\title{
Evaluating Optimal Phase Ordering in Obfuscation Executives
}

\author{
William Holder \\ School of Computing \\ University of South Alabama \\ Mobile, AL, USA \\ whd1102@jagmail.southalabama.edu
}

\author{
J. Todd McDonald \\ School of Computing \\ University of South Alabama \\ Mobile, AL, USA \\ jtmcdonald@southalabama.edu
}

\author{
Todd R. Andel \\ School of Computing \\ University of South Alabama \\ Mobile, AL, USA \\ tandel@southalabama.edu
}

\begin{abstract}
Obfuscation is a software protection technique that aims to increase the difficulty and amount of resources required to understand programs from the perspective of a malicious end user. The order and number of obfuscating transformations is determined by an obfuscation executive and the optimal arrangement of transformation defines the phase ordering problem. In this paper, we report on a case study evaluation for determining the optimal phase ordering for an obfuscation executive. We analyze obfuscation effectiveness of variants generated by Tigress, a dynamic virtualizing obfuscator with four transformation types. We test the evaluation of multiple orderings against a symbolic virtual machine to determine the strengths and weaknesses of each combination. We use overhead (cost) and effectiveness as the tradeoff space to determine the best sequence and ordering of transformations within this context. Our results show that, ideally, applying control flow transformation, data encoding, abstract transforms, and then dynamic virtualization provides the highest effectiveness on average against symbolic execution attacks.
\end{abstract}

CCS Concepts -Security \& Privacy $\rightarrow$ Software and application security; Software reverse engineering;

Keywords Obfuscation, software protection, symbolic execution, phase ordering, obfuscation executive

\section{Introduction}

Software has become larger and more complex with the development of new high level languages and improvement of commonly available computer hardware. Some recent estimates [1] state that programs such as Windows Vista, Facebook, and the Mac OS contain above 50 million lines of code, while the average

Permission to make digital or hard copies of all or part of this work for personal or classroom use is granted without fee provided that copies are not made or distributed for profit or commercial advantage and that copies bear this notice and the full citation on the first page. Copyrights for components of this work owned by others than ACM must be honored. Abstracting with credit is permitted. To copy otherwise, or republish, to post on servers or to redistribute to lists, requires prior specific permission and/or a fee. Request permissions from Permissions@acm.org.

SSPREW-7, December 4-5, 2017, Orlando, FL, USA

(C) 2017 Association for Computing Machinery.

ACM ISBN 978-1-4503-5387-8/17/12 ..\$15.00

https://doi.org/10.1145/3151137.3151140 high end car has about 100 million lines of code. More lines of code typically imply more complexity, which may lead to a greater number of security vulnerabilities. Besides the normal risk associated with software vulnerabilities, companies also store information inside of the program that they would prefer to be kept from the end user. This includes but is not limited to license key checks, fingerprints associating the user to the program, and proprietary algorithms.

These secrets are vulnerable to Man-At-The-End attacks, where malicious adversaries are able to monitor a program by inspecting the software and hardware of the system that it runs on [2]. Software companies may not want to deal with the headache of providing services like hosted-software over the Internet, and likewise clients normally prefer the convenience of running the program on their own system. Instead, companies can decide to use obfuscating transformations to protect program secrets.

Program obfuscation is directed automatically by an obfuscation executive, which determines the transformations used on the program along with how many times they will be performed. The ideal goal of the obfuscation executive is to create a transformed version of the program that when observed by a MATE attacker, gives as much information away about its protected secrets as a black box view of the original program. Complete protection of the obfuscated program is not possible however, because the secrets and semantics of the original program are still needed to run the obfuscated program. Instead obfuscation executives use transformations that increase the time and resources necessary to uncover secrets from the program. Unfortunately, this method of protection increases the time and space needed to run the program due to the added complexity, and with programs that have 50 million lines of code it is important that the transformations do not pass a certain time and space threshold while still protecting program secrets.

With these problems in mind, this paper provides a framework for determining the optimal phase ordering of an obfuscation executive for four distinct types of transformations. Our case study examines the effect an obfuscation executive's phase ordering has on the cost of running the obfuscated program along with its effectiveness in protecting its secrets, which will help determine which combination of transformations contributes the most to security and efficiency. Our findings confirm and illustrate other prior results that consider symbolic execution attacks, while as a contribution, providing a more in-depth analysis of optimal transformation ordering for the major categories of obfuscation transformations. In the rest of paper, we 
provide background and related work on the phase ordering in section 2, describe our case study methodology in section 3, provide results of our experiments in section 4, and give conclusions and future work in section 5 .

\section{Background}

In this section, we define the software attack model and the powers of malicious adversaries with direct access to executable code. We briefly review major categories of obfuscating transforms and the role of the obfuscation executive. We define our measurement framework and metrics for obfuscation strength and their relationship to the phase ordering problem. Our study is not the first to consider security of obfuscation in relation to transformation ordering, but does provide novel analysis in key respects to prior studies. Thus, we provide in section 2.7 an analysis of related research studies and the distinction of our case study analysis.

\subsection{Software Attack Model}

Obfuscation is primarily focused on defending distributed software from attacks where the adversary has full access to an executable and its environment. These attacks are referred to as Man-At-The-End (MATE) attacks, in contrast to Man-In-TheMiddle (MITM) attacks where the communications between two applications are intercepted. MATE attacks target the application as an executable file or during runtime in order to obtain any vulnerable information as well as make alterations to its behavior. Defending against MATE attacks is difficult because control of the environment and executable that the distributor is trying to protect belongs to the attacker after distribution. [2]

MATE attacks can be categorized in three categories: analysis, tampering, and distribution [3]. The targeted qualities of an executable that are of interest depends on the goal of the attacker, but all MATE attacks require some sort of analysis of the executable, excluding blind redistribution of the program. Proprietary algorithms are often the target of program analysis, which if discovered could put an algorithm that was time consuming and costly to develop into the competitor's hands for free. Attackers are often interested in tampering with security measures such as license checks as well. When the attacker has gained enough information on the application they know where to tamper with it to gain access to a regulated behavior. Alterations such as this allow for redistribution of the application, which can affect its sales. Distributors often uses methods of watermarking and fingerprinting that allows them to prove that they are the author of the application and identify the user that the application was given to. These however can still be bypassed by comparing two versions of the same application to discover fingerprints and alter the program in hopes of destroying a watermark. In this study we focus on two properties of interest to an adversary: a license key check and a proprietary algorithm.

\subsubsection{License Keys}

License keys are given to users who legitimately purchase a program, so that the program cannot be redistributed for free. These keys are validated by a license check written in the program, which prevents the program from running specific features if the key is not approved by the algorithm. Without protection these key checks are vulnerable to tampering that could allow skipping these checks altogether. Analysis of the license check could even allow the construction of key generating program not approved by the creators. Some license key checks can blacklist keys that are known to be obtained without purchase, and some require an internet connection to be validated such as most Windows operating systems [4]. In this paper, we report only on basic key checking algorithms.

\subsubsection{Proprietary Code}

While key checking algorithms are important for preventing a loss of sales from pirated software, other proprietary algorithms in the program may be just as important to protect. These algorithms can be encryption schemes you wish to keep secret, or an efficient picture altering algorithm that is better than their competitors. Some of the proprietary algorithms created by software developers took a large amount of time and money to create, and if an adversary were to discover said algorithm they could apply it to their own program for free.

\subsection{Obfuscating Transformations}

An obfuscation transformation $T$ converts a program $P$ into another program $\dot{P}$, where $P=T(P)$, and both $P$ and $P$ have the same observable behavior experienced by the user. Theoretically and practically, no transformations are known to completely prevent MATE attacks and we assume that given enough time and resources along with access to the system, a dedicated hacker may eventually discover program secrets. Instead the aim of these transformations is to obscure protected information from the attacker's view by increasing the amount of time and resources it will take to uncover it. Transformations are typically categorized along four lines: abstract, data, control, and dynamic [3].

\subsubsection{Abstract Transformations}

Abstraction transformations change the organization of the functions, modules, and classes in the program. By breaking these abstractions, it becomes difficult to identify the purpose of these structures. A function could be split into two segments, or two functions could be combined into one, which can confuse an adversary looking for one vulnerable algorithm.

\subsubsection{Data Transformations}

Data transformations replace data structures in the program with others that reveal less information. These transformations may take a constant value and convert it into a variable accessible through pointers, transform data into another format mathematically until it is needed by the program, or even replace mathematical expressions with more complex equivalents. These data transformations make it harder on the adversary so that observing the memory of the program does not leak sensitive data.

\subsubsection{Control Flow Transformations}

Control flow transformations modify the control flow structure of the program in order to hide which paths the program will take at runtime. One example of this is the addition of opaque predicates. An opaque predicate is a Boolean expression where the evaluation is known at compile time by the programmer, but not necessarily known to the attacker at runtime. Such predicates give the illusion 
of separate control flow paths when in reality only one of them ever executed. Control flattening takes each basic block in a program and converts the control flow into an infinite loop inside of a switch statement. By doing this, extra effort has to be made in order to identify the order that the blocks are executed.

\subsubsection{Dynamic Transformations}

Dynamic transformations introduce process-level virtual machines (PVM) into code and are categorized as static PVM or dynamic PVM. PVMs can be considered a much more complicated version of control-flow flattening, in which the original program's assembly instructions are converted into a different bytecode. This bytecode can only be understood by the interpreter, which is embedded in the original program and executes the program the same way it originally would have been executed. Interpreters usually contain their own virtual stack and registers. PVMs force the adversary to map the bytecode to its interpretation in order to discover the actual program. [5-6]. Dynamic PVMs change the interpreted instruction set architecture (ISA) continuously and randomly whereas static PVM techniques produce one ISA per program variant.

\subsection{Obfuscation Executives}

Protecting a program's content typically requires more than applying a single obfuscation transformation. Ideally a program should go through several transformations before it is safe enough for public access. Obfuscation executives are written to apply obfuscation transformations, and use algorithms to decide which transformations to use along with the order that they should be applied [3]. For example, Cohen [7] posed an algorithm that repeatedly adds transformations until the total space taken by the program exceeds the threshold determined by the user, as shown in Figure 1. Each transformation has a probability that it will be used, and a random number between 0 and 1 is determined as a minimum probability necessary. These transformations are used and their probability of use is added together until their sum is above the random number, afterwards if there is more space available for the program it will set the sum to zero, create a new random number, and applies more transformations. This technique ensures that there is enough diversity in each of the transformed programs produced.

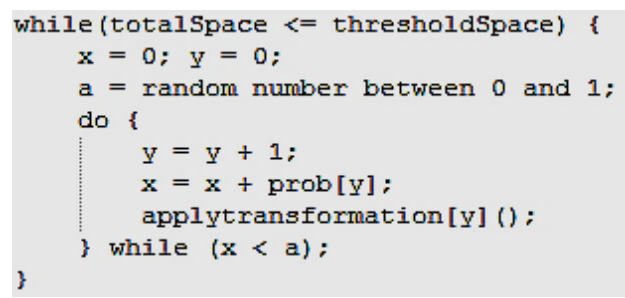

Figure 1. Cohen's Obfuscation Executive [3,7]

Cohen's technique is that it does not target protecting sensitive parts of the program and instead blindly adds transformations until there is no space left. Collberg, Thomborson, and Low developed an algorithm with this issue in mind $[3,8]$. This algorithm breaks the program into application objects, which is any program structure that can be obfuscated, shown in Figure 2. These objects are organized by the importance that they be protected, so that the obfuscation executive spends the most time protecting higher priority objects. The algorithm selects the highest priority object and applies the most appropriate transformation. It then updates that object's priority of protection based on how well the transformation protected it. It continues this cycle until either the maximum execution penalty is reached or the requested obfuscation amount is surpassed by the transformed program. Some weaknesses of this method is that the metrics for the obfuscation amount are hard to estimate, and the accepted cost may be reached before the desired obfuscation amount is reached. Also because individual parts of the program are being transformed, the internal data structures must be preserved to prevent data corruption or misuse. Heffner and Collberg further propose a finite state automaton (FSA) to track these data dependencies and prevent them from being broken [9]. The FSA contains all of the data dependencies between each transformation and, during obfuscation, the executive selects a path that will respect such dependencies.

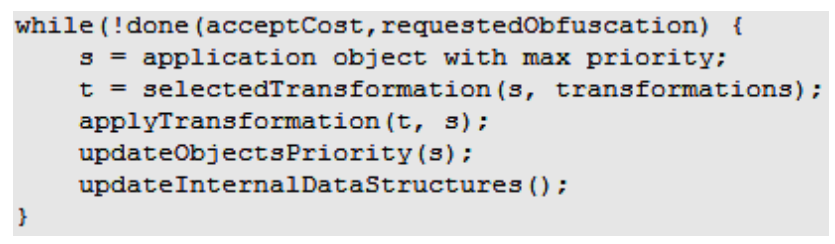

Figure 2. Collberg, Thomborson, and Low's Obfuscation Executive [3,7]

\subsection{Obfuscation Metrics}

Obfuscation metrics are used to estimate how well a program secret is protected by a transformation. Collberg and Nagra's definition pose several metrics for obfuscation strength [3] which include potency, resilience, stealth, and cost (overhead) [10]. In this study, we limit our measurements to effectiveness and overhead:

- Effectiveness captures the notion that good obfuscating transformations should increase the time or resources for the necessary analyses that reveal the secret property on the obfuscated program than it is on the original [3]. Let $T$ be an obfuscating transform, $P^{\alpha}$ be a program with property $\alpha, A$ be a program analysis that reveals property $\alpha$, and let $P^{\alpha}$ be an obfuscated version of $P^{\alpha}$ s.t. $T\left(P^{\alpha}\right)=P^{\alpha}$ and $A\left(P^{\alpha}\right)=\alpha_{\mathrm{d}}$. According to Collberg and Nagra [3], transformation $T$ is effective if $\alpha_{\mathrm{d}} \approx \alpha$ (analysis $A\left(P^{\alpha}\right)$ does not reveal the original property) and $A\left(\dot{P}^{\alpha}\right)$ requires more resources to compute than $A\left(P^{\alpha}\right)$. Likewise, transformation $T$ is ineffective if $\alpha_{\mathrm{d}} \approx \alpha$ (analysis $A\left(\dot{P}^{\alpha}\right)$ reveals the original property) and $A\left(P^{\alpha}\right)$ requires the same amount of resources to compute as $A\left(P^{\alpha}\right)$. Transformation $T$ is defective if $\alpha_{\mathrm{d}} \approx \alpha$ (analysis $A\left(P^{\alpha}\right)$ reveals the original property) and $A\left(\dot{P}^{\alpha}\right)$ requires less resources to compute than $A\left(P^{\alpha}\right)$.

- Potency takes into account that an adversary may have more than one analysis that they may apply to an obfuscated program. Based on the definition in [3], we consider a 
transformation $T$ potent relative to a set of analyses $A$ and obfuscated program $P^{\alpha}$ (transformed by $T$ ) if there exists at least one $A_{i} \in A$ such that $T$ is effective w.r.t. $P^{\alpha}$ and analysis under $A_{i}$, and for all other analyses $A_{j} \in A$ (not equal to $A_{i}$ ), $T$ is not defective with respect w.r.t. $P^{\alpha}$ and analysis under $A_{j}$. To summarize, a potent obfuscating transformation makes at least one analysis harder and does not make any other analysis easier.

- Cost (overhead) is a measure of cost in memory, runtime speed, or response time, perceived quality, etc. added by a transformation [10]. Because of the added complexity that most obfuscation transformations induce, performance decreases compared to the original program. Certain transformations such as scrambling variable names and removing formatting induce no overhead. Changes that increase potency will add extra complexity and most likely take more time and space to run.

\subsection{Adversarial Analysis}

Static and dynamic analyses are two major categories of program analysis vectors used by MATE attackers to determine program secrets. Programs such as Radare 2 and IDA Pro can be used for both static and dynamic analysis [12]. Debuggers such as gdb can be used to place breakpoints in a program and read active instructions, while objdump can be used to print the symbol table and assembly of an executable [13-14]. Symbolic execution tools such as KLEE can be used to automatically generate tests for input into the program [15-16]. Symbolic execution is similar to fuzzing except instead of trying every combination of input it instead keeps a set of all the values that can exist past each branch in the code. Memory analysis tools such as Linux Memory Extractor (LiME) can be used to peer into process space memory [17]. A few papers are listed below explaining how to accomplish analysis on transformations such as virtualization and data obfuscation.

Adversarial program analysis is conducted for legitimate purposes when reverse engineering malware. Often automated tools fail because of transformations that are targeted at antiemulation, anti-debugging, anti-disassembly, or antidecompilation. Semi-automated or manual techniques have been used to get past these failures. Rolles, for example, created a method for analyzing virtualized malware [6] and deals with the assumption that hidden code is visible and unprotected in memory when the program executes. He observes that malware finds ways to hide the code such as using multiple executables that debug and modify the original.

Deobfuscation is a general technique to reduce complexity of protected programs. Yadegari et al. propose a general approach to deobfuscating executable code that can be applied even newly undiscovered transformations [18]. The technique uses taint propagation and semantic preserving code transformations while keeping as few assumptions as possible about any specific obfuscation technique used. Their study reports favourable results for reducing complexity in program variants created by Themida, Code Virtualizer, VMProtect, and Execryptor.

\section{Problem Statement and Related Work}

The purpose of this paper is to bring to light the phase ordering problem: the fact that the overall effect of transformations can cause an obfuscated program to be either more or less resilient to attack based on the ordering used. We discuss this key concept briefly and provide a review of related work where obfuscation effectiveness and ordering has been considered.

\subsection{Phase Ordering Problem}

Phase ordering describes the operation of an obfuscation executive in selecting which transformations and in what order they are applied. Given a set of transformations, an executive may apply a transformation more than once or not apply it at all. A poor ordering can damage the potency or effectiveness of the obfuscated program while still increasing the cost of the program. Furthermore, obfuscating transformations can destroy other protections designed to watermark or fingerprint a program [3]. For example, a watermarking method for android apps may be based on events called in the application [11]. If the events were split by abstraction obfuscation, or the data and control flow were altered, it is possible that the watermark would be destroyed in the process. We conduct a case study experiment to evaluate the ordering effectiveness and efficiency for a well-known obfuscator and consider attacks based on symbolic analysis from a wellknown analysis tool. Section 4 details the approach we use and section 5 reports the results.

\subsection{Prior Studies}

There are historically few studies that attempt comparison of obfuscators or transformation techniques themselves. Macbride et al. studied the effects of transformations such as class/function renaming, restructuring control flow, and string encryption [20] on sorting algorithms such as QuickSort, BubbleSort, and RadixSort. The execution cost was measured to determine the overhead associated with each of these transformations versus the resulting complexity of the code. Complexity was measured by the number of IF, GOTO/LABELS, WHILE, and FOR statements in the program. It was determined that performance loss increased when the complexity of the code increased. Macbride's study only used KlassMaster and Dasho-Pro obfuscators on Java source, but illustrates evaluation of program complexity.

Ceccato et al. conducted an empirical study on the potency of obfuscation transformations created by the Java obfuscation tool SandMark [21]. In their study, $22 \mathrm{PhD}, 39$ Master, and 13 Bachelor students with good knowledge on Java programming were given these transformed programs and evaluated on the correctness, time, and efficiency it took to either analyze or attack the program. Correctness was measured by their ability to perform the task given, time by how long it took to complete the task, and efficiency by the number of correctly performed tasks in a minute. Overall it was shown that adding any of the transformations increased the time and efficiency of completing the tasks while decreasing the number of correctly performed tasks.

The phase ordering problem is similar to phase ordering in optimizing compilers, where transformations in the wrong order can actually destroy the improvements of the first transformation. 
In [19], Qu'eva studied the phase ordering problem in compilers. He highlights the fact that an infinite amount of optimization orderings exist, which leaves finding the most optimal transformation sequence uncertain. His study compares optimizing algorithms in relation to the power consumption, size, and computation time of the program. We follow the model of cost metrics in our study for measuring phase ordering.

Banescu et al. provide the most recent and comprehensive study with insight to the phase ordering problem that we are aware of [26]. Their work primarily characterizes the resilience of obfuscating transformations against automated symbolic execution attacks. They evaluated over 5000 different $\mathrm{C}$ programs using implementations of known transformation techniques provided by the Tigress C obfuscator [23] and ObfuscatorLLVM [27]. Similar to our study, they run symbolic analysis on 48 $\mathrm{C}$ programs with embedded secrets that are unprotected, and then run the same symbolic analysis on obfuscated variants of each original program. Banescu et al. use 30 different configurations of Tigress and 9 different configurations of Obfuscator LLVM while using KLEE [15] and angr [28] for symbolic analysis.

Their results, although focused primarily on showing that symbolic execution attacks are wildly successful even on protected programs, partially illustrate the effects of executive ordering. As our contribution in this paper, we focus on this ordering exclusively and go beyond pairing of individual transformations or application of the same transformation twice by considering the permutations of the four basic categories of transforms (abstract, data, control, dynamic). In contrast, 1) we do not use a second obfuscator (Obfuscator LLVM) or a second symbolic analyzer (angr), 2) we evaluate 132 variant programs from 6 random programs generated by Tigress with 6 different password/code lengths versus a predetermined benchmark set of $48,3)$ we consider permutations of 3 and 4 transformation categories vs. only two at a time, and 4) we use multiple transformations of the same category type that are provided by Tigress vs. considering them individually. We discuss how our results confirm and differ from those in [26] in section 6 .

\section{Experimental Framework and Case Study}

Our case study determines optimal phase ordering using implementations of known transformation algorithms provided by Tigress [22]. We perform all experiments on a Dell Precision T5600 workstation with two Intel Xeon E5-2630 processors (Six Core $2.2 \mathrm{GHz}$ ) and $128 \mathrm{~GB}$ RAM, running Ubuntu 14.04 operating system in a Docker container under Windows 7. As our contribution, the results of our case study provides: 1) a general framework for evaluating any obfuscator that provides finegrained control of individual obfuscating transformations (abstract, control, data, and dynamic); 2) recommendations for optimal phase ordering in obfuscation executives; and 3) analysis results based on applying our framework to the Tigress obfuscator. Our framework consists of several key steps, delineated in the following subsections: 1) selecting benchmarks, 2) choosing the obfuscation engine, 3) choosing transformation algorithms, 4) choosing metrics, 5) choosing analysis set, and 6) performing the variant generation and analysis.

\subsection{Benchmark Program Selection}

As the first step of our framework, benchmark programs must be chosen to evaluate. We use random sample programs that are generated by the Tigress software. Figure 3 illustrates the main method for a sample random program and Figure 4 illustrates a function with secret information that needs to be protected from a malicious adversary. Each random program can potentially have two embedded secrets: a password that is entered by the user at runtime and an activation code that is provided as a command line argument. We utilize two types of random programs for our case study: 1) three with just a password and 2) three with a password and activation code. For password-only programs, we generate random programs (pass) with varying lengths of 6,9 , and 12 characters and, for password/code programs (pass/code), we generate random programs with three different combinations of lengths, as table 1 depicts. Figure 5 depicts the Tigress program generation command.

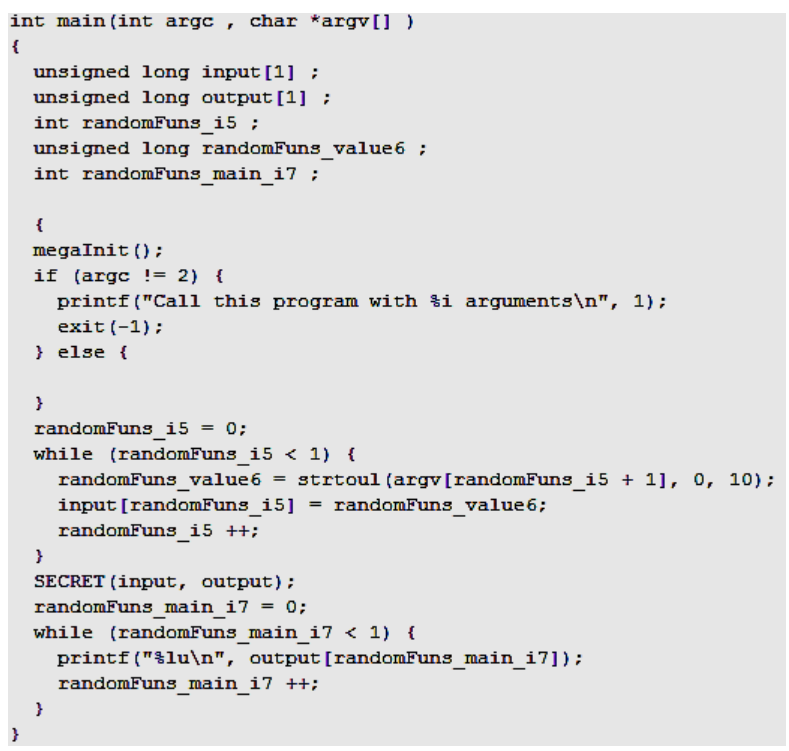

Figure 3. Example main () function generated by Tigress

\subsection{Transformation Engine}

Tigress has been used to measure the stealth, resilience, and code stylometrics of obfuscated code in other separate studies [2325]. It is controlled through terminal input by the end user, and is developed for Linux and Mac OS. For our study, we choose transformations manually, acting as an obfuscation executive, in order to test different combinations of transformations.

Table 1. Original program types with password/code

\begin{tabular}{|c|c|c|}
\hline Type & Password & Code \\
\hline pass-6 & ! @ \# \$ㅅ & - \\
\hline pass-9 & 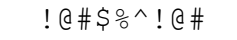 & - \\
\hline pass- 12 & 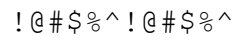 & - \\
\hline pass $-3 /$ code 2 & ! @ \# & 12 \\
\hline pass $-3 /$ code 3 & $\# ! \frac{\circ}{0}$ & 234 \\
\hline pass- $4 /$ code 4 & Q \#\#! 응 & 1234 \\
\hline
\end{tabular}




\subsection{Transformation Algorithms}

We use the follow Tigress transformations in our case study:

- Abstract: merging, splitting, random argument addition

- Data: encoding literals, encoding data, encoding arithmetic

- Control: control flattening, opaque predicates

- Dynamic: process level virtualization

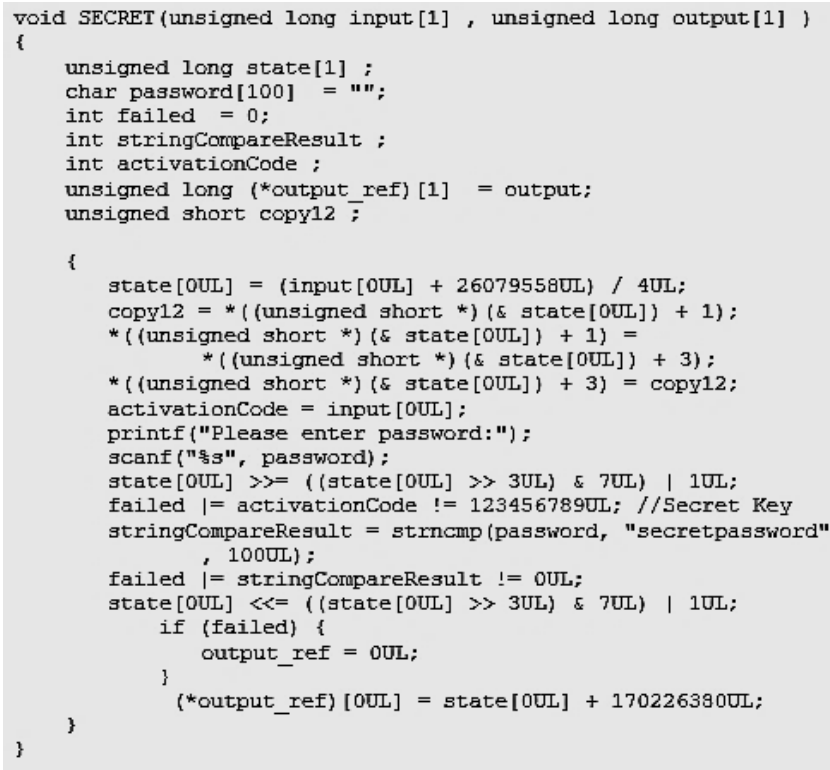

Figure 4. Example SECRET ( ) function generated by Tigress

tigress --Verbosity=1 --Seed=0 --Transform=RandonFuns
--RandomFunsName=SECRET --RandomFunsType=long
--RandomFunsInputSize=1 --RandomFunsStateSize=1
--RandomFunsOutputSize=1 --RandomFunsCodeSize=10
--RandomFunsActivationCodeCheckCount=1
--RandomFunsActivationCode=123456789
--RandomFunsPasswordCheckCount=1
--RandomFunsPassword=secretpassword
--RandomFunsFailureKind=segv
--out=example.c empty.c

Figure 5. Random program generation options in Tigress

Tigress abstract transformations alter the organization and structure of the functions to hide what they actually do. The merging transformation takes two functions and combines them into one. The splitting transformation takes two functions and splits them into separate pieces. Random argument addition adds bogus arguments to a function to imply there is an extra data dependency when there is not. We use these three abstract techniques, which can increase the potency of the program by repeatedly splitting, merging and adding extra parameters to functions [22].

Tigress data transformations give alternate representations of data and data structures. Encoding the literals of the program are expected to be useful in hiding the generated program's key check, due to the fact that it compiles as plain text in the read only memory section. Data encoding hides data values until they are used as well, so that memory analysis alone will not suffice in uncovering sensitive information. Encoded arithmetic hides how the data is being manipulated so that the behavior of an algorithm is not straight forward. We use these three data techniques, which are expected to increase analysis time and resources for finding the key in sample programs [22].

We use two Tigress control flow transformations: control flow flattening and opaque predicate conditional statements. Both of these techniques are used to break up basic IF, FOR, and WHILE statements so that control flow of the program is not obvious from observing the executable, mainly by combining basic blocks into a large switch statement, with a value identifying which case statement to execute next. Opaque predicates will mislead the analyzer by doing unnecessary or misleading checks to determine the control flow path, which may never take its alternate path. These techniques protect against static analysis well, and require dynamic analysis to determine which paths will be executed during a legitimate run-through of the program [22].

The only dynamic transformation that we experiment with is static process level virtualization. Tigress creates a unique bytecode and interpreter in order to run the program, which makes static and dynamic analysis difficult. Program understanding for virtualization requires examination of the bytecode and how it interacts with the interpreter at runtime. Tools such as Radare2 can be used to analyze the interpreter and read the assembly instructions both statically and dynamically, whereas the virtual stack has to be examined using a memory analysis tool such as LiME [22] to uncover the data hidden by the program.

\subsection{Analysis Methods and Tools}

For adversarial analysis of obfuscated program variants, we use the following tools: Radare2, gdb, objdump, and KLEE. We conduct manual analysis on variants using Radare 2 and gdb for dynamic analysis objdump for static analysis. For automated analysis, we use KLEE to perform symbolic execution on variants and derive a set of user inputs that allow the program to perform properly. We attempted to follow Rolle's method to attack static virtualization [6] and utilize general deobfuscation techniques from [18]. Automated analysis was performed in a Docker container with a KLEE environment setup inside. We use a Perl script to parse the output created by KLEE every five seconds until a success code is recovered, in which a test ends and the total run time of the program is recorded. We use the time command, native to Linux, to output the execution time for automated tests.

\subsection{Test Program and Variant Generation}

We divide our case study into three parts. Using randomly generated programs from Tigress (designated as $P$ ), we create variants using Tigress obfuscation options based on a specific sequence of one or more transformations. In part 1 of our study, we generate three different variants of an original program: one using control flow transformations, one using abstract transformations, and one using data transformations. Each of these variants are tested individually for its cost and efficiency compared to the original program. Figure 6 illustrates the three programs $\left(P^{C}, P^{A}, P^{D}\right)$ generated after applying each transformation. In part 2 of or study, we take the original program and apply all permutations of these three transformation 
categories to create an obfuscated version. Six different programs are generated in this manner, one for each different enumeration of the transformations. We then evaluate these variants against the original in regards to cost and efficiency. The six resulting programs $\left(P^{C A D}, P^{A C D}, P^{D A C}, P^{C D A}, P^{A D C}, P^{D C A}\right)$ are illustrated in Figure 7.

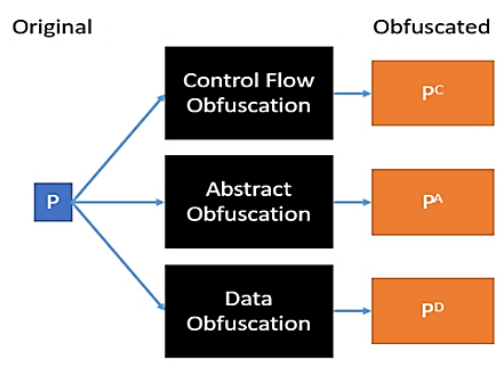

Figure 6. Part 1- Single Application of Abstract, Data, and Control Transformations
Original

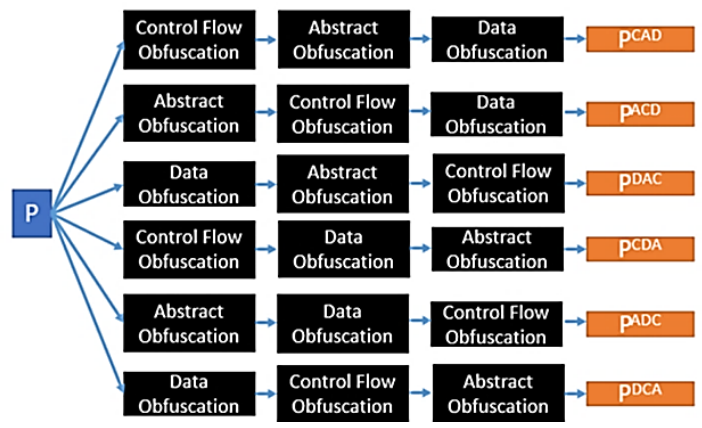

Figure 7. Part 2 - Permutations of Abstract, Data, and Control Transformations

In part 3, we take the same steps for permutations as in part 2 , except we consider the use of dynamic obfuscation using virtualization. We generated 6 versions where virtualization is added first and 6 versions with virtualization added last. We opt to not include dynamic transformations into the enumeration in part 2 in order to avoid increasing the number of programs that need to be examined and because of the difficulty of breaking virtual obfuscators. We evaluated each of these programs for its cost and efficiency compared to the original program. The resulting 12 programs $\left(P^{V C A D}, P^{V A C D}, P^{V D A C}, P^{V C D A}, P^{V A D C}, P^{V D C A}, P^{C A D V}\right.$, $\left.P^{A C V D}, P^{D A C V}, P^{C D A V}, P^{A D C V}, P^{D C A V}\right)$ are illustrated in Figure 8. Based on the 6 original program types which have varying lengths of password and activation codes (seen in table 1), we generate 21 variants of each program during part 1,2, and 3 of the case study. As a result, we analyze 132 programs including the originals.

\section{Results}

For each variant, either one or two pieces of information are required to "crack" the software: a password and an activation code (seen in table 1). For symbolic execution, KLEE must be set to expect a certain number of characters for the password and a certain number of digits for the activation code. For efficiency, the case study emphasizes the impact that large input sizes have on the length of time required for KLEE to recover the successful inputs. Success is determined when the program outputs a success code in stdout. Results for KLEE analysis time for the passwordonly programs are seen in Figure 9 and for password/code programs are seen in Figure 10.

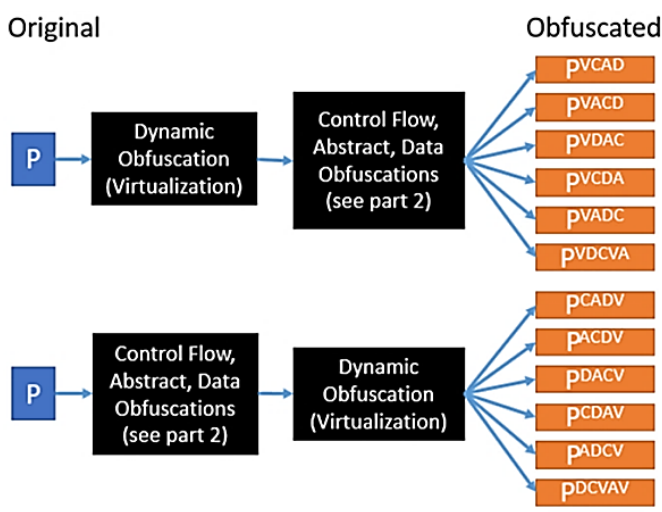

Figure 8. Part 3 - Permutations of Abstract, Data, Control Transformations with Virtualization

\begin{tabular}{|l|r|r|r|}
\hline Cfile & \multicolumn{1}{|l|}{$\begin{array}{l}\text { Six } \\
\text { Characters }\end{array}$} & \multicolumn{1}{l|}{$\begin{array}{l}\text { Twelve } \\
\text { Characters }\end{array}$} \\
Characters \\
\hline original & 5.106 & 5.096 & 5.053 \\
\hline A & 5.075 & 5.073 & 5.05 \\
\hline C & 5.09 & 5.078 & 5.05 \\
\hline D & 5.094 & 5.099 & 5.054 \\
\hline ACD & 5.083 & 5.076 & 5.05 \\
\hline ADC & 5.073 & 5.072 & 5.051 \\
\hline CAD & 5.051 & 5.05 & 5.05 \\
\hline CDA & 5.055 & 5.051 & 5.051 \\
\hline DAC & 5.09 & 5.094 & 5.051 \\
\hline DCA & 5.096 & 5.072 & 5.051 \\
\hline ACDV & 5.05 & 5.05 & 5.051 \\
\hline ADCV & 5.051 & 5.055 & 5.054 \\
\hline CADV & 10.052 & 10.099 & 5.054 \\
\hline CDAV & 15.057 & 10.099 & 10.052 \\
\hline DACV & 5.094 & 5.069 & 5.052 \\
\hline DCAV & 10.096 & 5.092 & 5.052 \\
\hline VACD & 10.052 & 10.092 & 5.051 \\
\hline VADC & 10.052 & 10.093 & 5.052 \\
\hline VCAD & 10.053 & 10.093 & 5.057 \\
\hline VCDA & 10.074 & 5.094 & 5.052 \\
\hline VDAC & 10.054 & 5.092 & 5.053 \\
\hline VDCA & 10.052 & $\mathrm{NA}$ & 5.053 \\
\hline
\end{tabular}

Figure 9. KLEE analysis time (in seconds) for password-only variants

\subsection{Part 1 (Single Transform) Analysis}

For all tests in part 1, KLEE was successful in obtaining the password/activation code necessary to recover the success code. Figure 11 show a comparison of the time required for each test to run compared to the original un-obfuscated program for password-only programs, while Figure 12 shows execution time for password/code programs. Figure 11 shows that obfuscation 
techniques used individually were either ineffective or defective, requiring either less time or the same amount of time as the original program to solve. The average time for the individual obfuscation tests overall were defective (seen in Figure 19). The average execution time for all tests were within 0.03 seconds, which could be a result of the 5 second check rate of the perl script written to check for success, therefore it may be more accurate to say that all tests were ineffective instead of defective.

\begin{tabular}{|l|r|r|r|}
\hline Cfile & $\begin{array}{l}\text { Three Char/Code } \\
\text { (4 character KLEE) }\end{array}$ & $\begin{array}{l}\text { Four Char/Code } \\
\text { (4 character KLEE) }\end{array}$ & $\begin{array}{l}\text { Three Char/Code } \\
\text { (6 character KLEE) }\end{array}$ \\
\hline original & 60.068 & 215.059 & 1300.112 \\
\hline A & 110.075 & 35.083 & 870.093 \\
\hline C & 15.072 & 40.067 & 595.092 \\
\hline D & 75.07 & 215.064 & 4850.281 \\
\hline ACD & 115.085 & 40.079 & 1580.112 \\
\hline ADC & 115.071 & 35.064 & 1585.105 \\
\hline CAD & 25.11 & 50.058 & 3615.17 \\
\hline CDA & 55.08 & 70.074 & 755.077 \\
\hline DAC & 40.068 & 75.058 & 1045.09 \\
\hline DCA & 55.07 & 90.082 & 2020.123 \\
\hline ACDV & 285.082 & 155.073 & 4825.2 \\
\hline ADCV & 280.083 & 135.084 & 2780.161 \\
\hline CADV & 210.068 & 325.089 & 12970.491 \\
\hline CDAV & 205.091 & 360.085 & 6105.276 \\
\hline DACV & 110.069 & 195.079 & 2220.122 \\
\hline DCAV & 185.094 & 390.063 & 8360.305 \\
\hline VACD & 455.069 & 580.081 & 12280.421 \\
\hline VADC & 925.095 & 585.088 & 10400.34 \\
\hline VCAD & 465.072 & 575.077 & 5995.228 \\
\hline VCDA & 220.079 & 490.065 & 7295.261 \\
\hline VDAC & 460.072 & 575.069 & 7465.272 \\
\hline VDCA & 245.064 & 515.07 & 5655.224 \\
\hline
\end{tabular}

Figure 10. KLEE analysis time (in seconds) for password and code variants

The tests for password/code protected programs showed more varied results, with Abstraction and Control Flow obfuscations being defective and Data obfuscation being effective on average. An exception exists for Abstraction done on a three character password and three digit code, given a four character/digit length in KLEE. Another exception can be seen in the four character/digit inputs for Data obfuscation which is ineffective in increasing the time required to successfully recover the desired inputs. Figure 12 shows a more accurate representation of the strengths of each obfuscation since the length of time required to solve these programs are much greater than the check rate of the test script, as well as much greater than the password only programs.

\subsection{Part 2 (A/C/D Permutation) Analysis}

For all tests in part 2, KLEE was successful in obtaining the password/activation code necessary to recover the success code as well. Figure 19 shows that all orders of Abstraction, Data, and Control Flow obfuscation for single password programs were ineffective on average. Individually, the program with a twelvecharacter password was closest to the original time, with no increase/decrease in time as seen in Figure 13. The average times are off by 0.05 seconds at max, so although they are technically defective it would be more accurate to categorize them as ineffective.

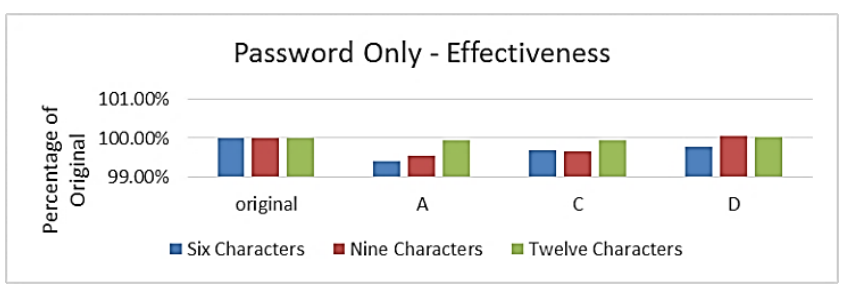

Figure 11. Part 1 effectiveness on password-only programs using single A,C,D transformations

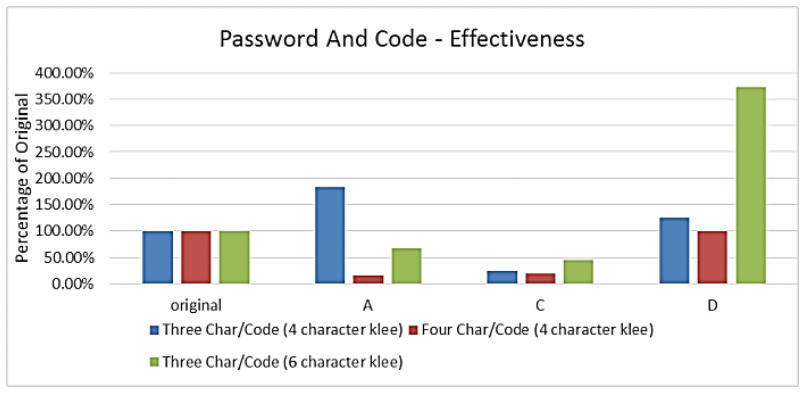

Figure 12. Part 1 effectiveness on password/code programs using single A,C,D transformations

Almost all password/code protected programs were improvements in that they increased the time required to recover the correct inputs for a success. Programs obfuscated in the order CDA and DAC on average were defective, while ACD, ADC, $\mathrm{CAD}$, and DCA were effective on average (seen in Figure 19). An outlier to these tests seen in Figure 14 would be the fourcharacter/digit test, where KLEE is set to expect inputs of size four characters. The four-character tests were all defective, which lowered their averages. Figure 14 also shows that CAD and DCA were only effective where KLEE was expecting a 6 character/code input, otherwise the tests were defective.

\subsection{Part 3 (A/C/D/V Permutation) Analysis}

For all tests in part 3 where virtualization came first, none of them were defective. Shown in Figure 19, the average time for all versions of the password only programs were effective, however Figure 15 shows that each obfuscation order has at least one test that was ineffective. Six-character password-only variants were all effective against symbolic analysis, and had almost a $200 \%$ increase in runtime, while twelve-character password programs were all ineffective. Obfuscation orders VACD, VADC, and VCAD had the largest improvement on average, taking above 8 seconds to run. Programs that took password/code inputs had a significant increase in runtime when obfuscation was added. Every test was effective, on average and individually. In Figure 16 VACD has the highest average increase in runtime, which was 73 minutes. VACD also had the highest runtime of all Virtualization first programs, which was 3 hours and 25 minutes that was from the 3 character/code and 6 character KLEE test. Figure 16 shows 
that all tests with virtualization first had above a $200 \%$ increase in runtime. It is important to note that since Figure 16 uses the original program as a benchmark for percentage slowdown, an $800 \%$ increase in the 3 character/code tests does not mean it took longer than a $200 \%$ increase in the 4 character/code tests.

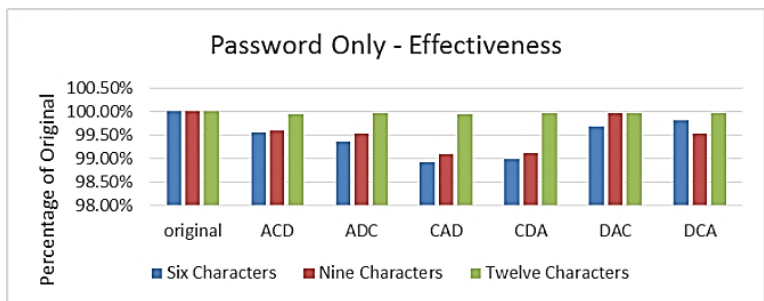

Figure 13. Part 2 effectiveness on password-only programs using permutations of A,C,D transformations

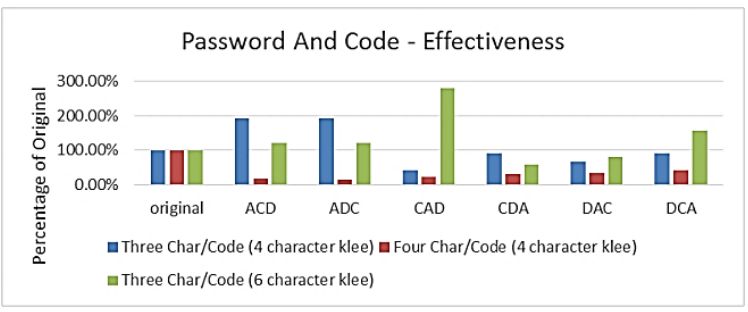

Figure 14. Part 2 effectiveness on password/code programs using permutations of A,C,D transformations

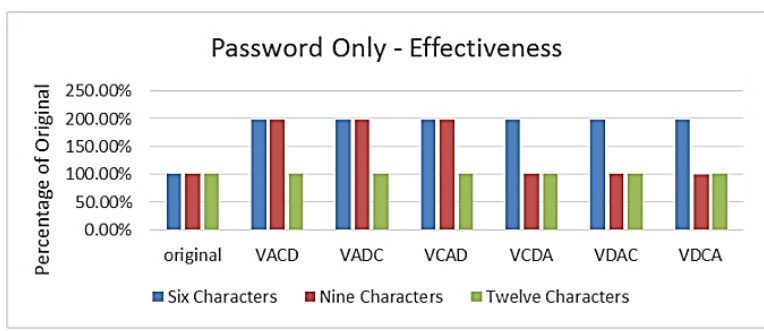

Figure 15. Part 3 effectiveness on password-only programs using permutations of $\mathrm{V}$ first with $\mathrm{A}, \mathrm{C}, \mathrm{D}$ transformation type

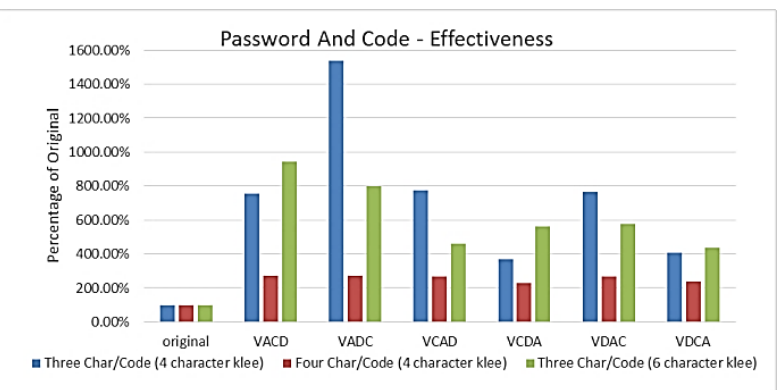

Figure 16. Part 3 effectiveness on password/code programs using permutations of $\mathrm{V}$ first with A,C,D transformations

Most tests for part 3 where virtualization came last were either effective or ineffective. None of the tests were defective for password only programs, however, as Figure 17 illustrates, only
CADV, CDAV, and DCAV were effective on average. CDAV was the only obfuscation order where all tests were effective, and took close to 11.5 seconds to complete on average. Figure 17 illustrates programs with twelve character passwords were all ineffective, which was a repeating pattern in all tests done overall.

Excluding 4 character/digit password protected programs, all other tests done on password and code protected programs were effective. In Figure 18 all averages are above the original programs time, with CADV having the largest average of 1 hour and 15 minutes. The largest individual time was also CADV for a 3 character/code protected program, with KLEE set to expect 6 character/digit inputs, and took a total of 3 hours and 36 minutes. The least effective order was DACV with an average execution time of 17 minutes. For 4 character/digit password protected programs, using an expected 4-character length in KLEE; ACDV, $\mathrm{ADCV}$, and DACV were defective as seen in Figure 18.

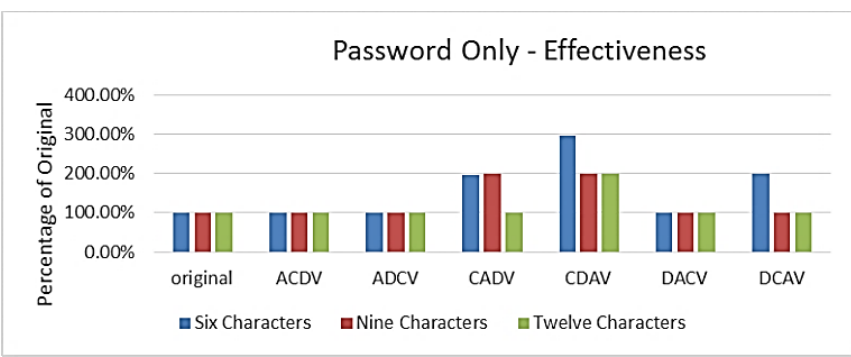

Figure 17. Part 3 results on password-only programs using permutations of V last with A,C,D transformations

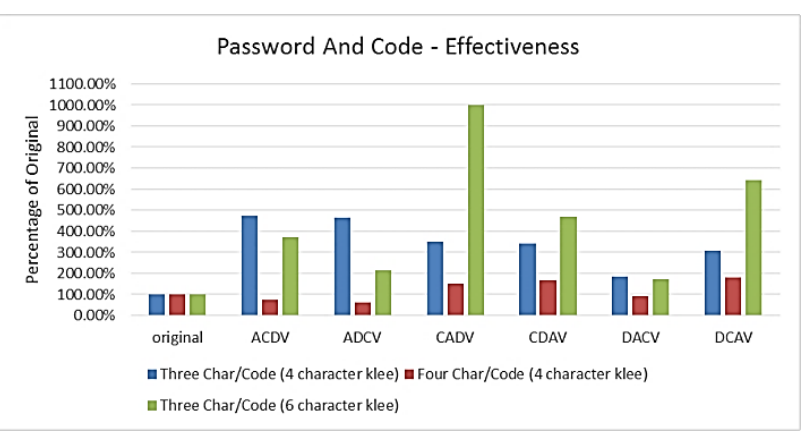

Figure 18. Part 3 results on password/code programs using permutations of $\mathrm{V}$ last with A,C,D transformations

\subsection{Password-Only vs. Password/Code Analysis}

Overall, for password-only programs the virtualized transformation showed an increase in analysis time. Figure 19 shows that ACDV, ADCV, and DACV did not have any increase in effectiveness compared to the original. Along with this, twelvecharacter passwords showed no improvements in any of the transformation combinations used as seen in Figure 19 except for in CDAV. CDAV had the highest average because of this, taking an average of 11.5 seconds to complete. The largest increase in analysis time for password/code protected programs on average was 1 hour and 15 minutes, as seen in Figure 20, while the highest increase in runtime relative to the original was the VADC test for 
3 character passwords and digit code, where KLEE was set to 4 characters/digits, was a $1500 \%$ increase as seem om Figure 20. Password/code protected programs on average showed above a $200 \%$ increase in effectiveness for Data transformations in part 1 , $\mathrm{CAD}$ in part 2, and all virtualized programs except for DACV in part 3. Orderings with defective averages include $\mathrm{A}$ and $\mathrm{C}$ from part 1, and CDA and DAC in part 2. Other transformation orderings where all tests were effective were CADV, CDAV, DCAV, VACD, VADC, VCAD, VCDA, VDAC, and VDCA (which all use virtualization as a transformation method).

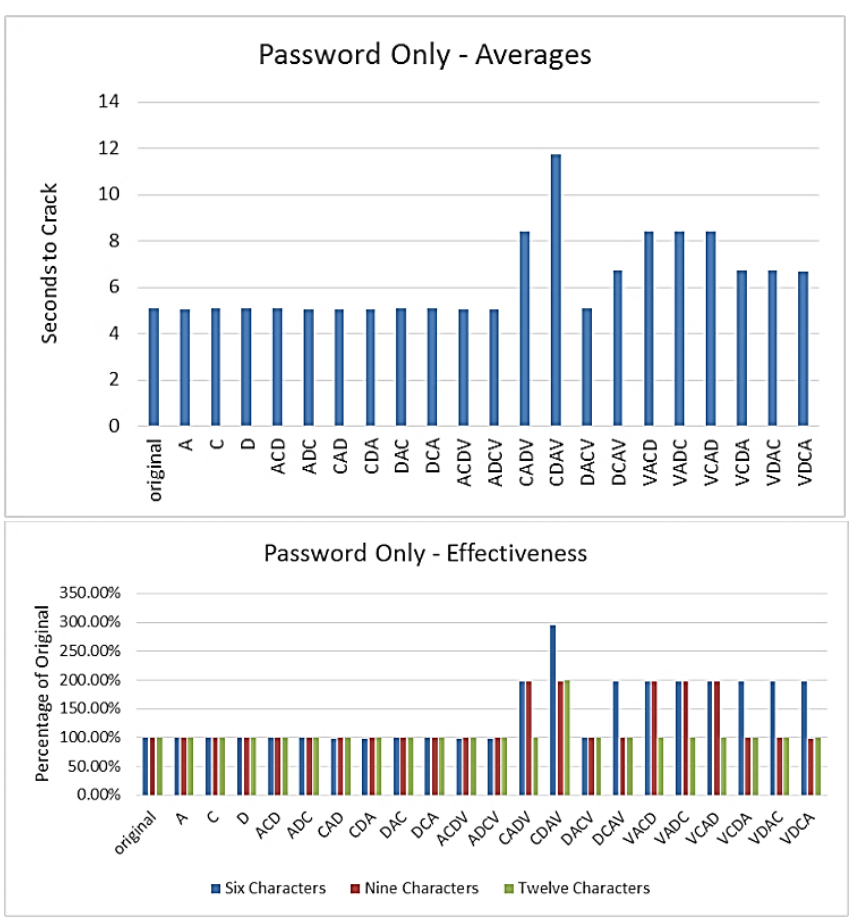

Figure 19. Password-only program analysis summary

\subsection{Effectiveness and Overhead}

Based on the results of our case study analysis, we conclude that programs with password-only protection had the least success in increasing effectiveness against adversarial symbolic analysis. It is important to note that the perl script written to check for a successful run of the program had a refresh rate of 5 seconds, therefore the analysis may have finished sooner than the results suggest. The original programs for all password lengths saw a maximum increase of $300 \%$, however this was only an increase of 10 seconds. This means that programs with only one input have little benefit from using obfuscation. Another important detail is that the original analysis runtime was 5 seconds long for all input lengths, which suggests that programs with short runtimes do not benefit from obfuscation. This can be reinforced by comparing averages of password-only and pass/code programs, as seen in Figure 21. Password and code programs have a much longer runtime both in the original program and after transformation.

Observing tests where the input size KLEE is set to expect is equal to the size of the password and/or code showed the most defective and ineffective results as seen in Figure 19 and 20. For password-only programs, KLEE was set to expect passwords of length 12 or below. In programs with 12 character passwords, all tests except for CDAV were ineffective as opposed to other password lengths. Figure 19 illustrates that as the password gets longer, the tests are less effective compared to the original. This could mean that knowing the password and code length ahead of time decreases the length of time KLEE needs to recover the password and code. To further support this point, a four character password and four digit code was tested with KLEE when set to expect both 4 character passwords and 4 digit codes or less. The results shown in Figure 20 shows that only CADV, CDAV, DCAV, VACD, VADC, VCAD, VCDA, VDAC, and VDCA were effective. 4 character/digit tests had the least benefit from obfuscations compared to both 3 character/digit tests. This is especially interesting when comparing 3 character/digit protection to 4 character/digit protection since efficiency was higher for lengths of 3 compared to lengths of 4 . This is a comparison of efficiency to the original program however, 4 character/pass programs still took longer to run than 3 character/pass programs.

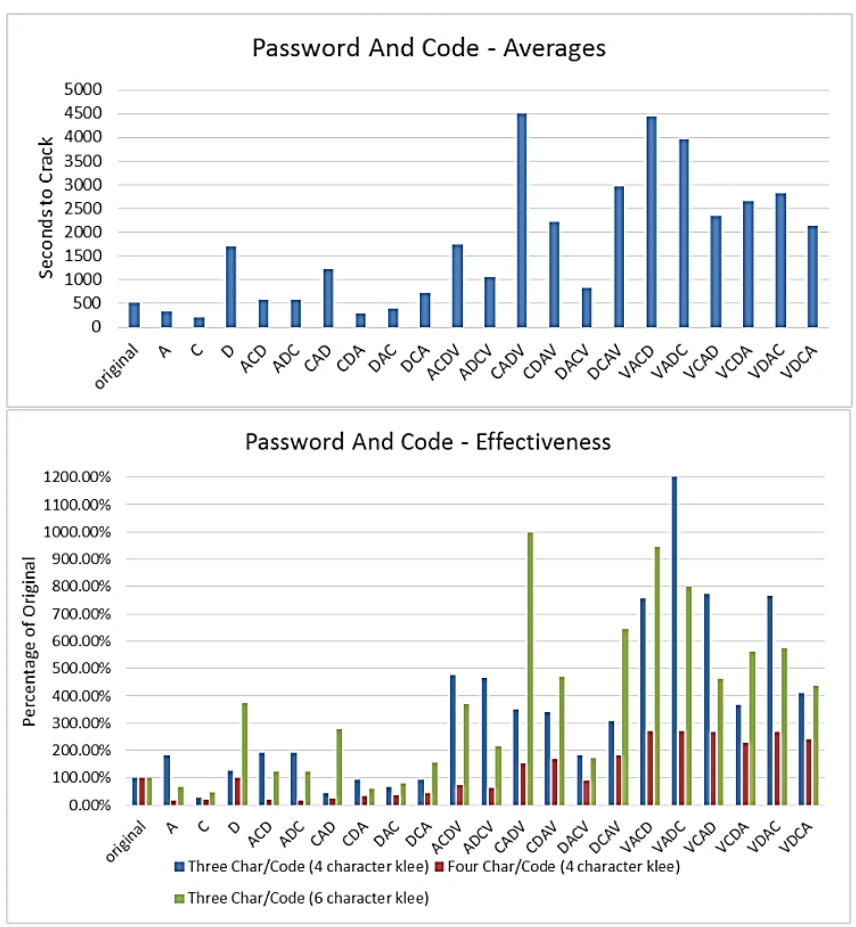

Figure 20. Password/code program analysis summary

We calculated overhead of all obfuscated variants based on the size of the assembly code (used by KLEE) compared to the original program. Figure 22 summarizes the average overhead for all program variants by ordering and also shows overhead just for password/code programs, which is slightly higher. The highest average overhead values are from virtualization-first variants (around $100-110 \%$ for all programs and $113-128 \%$ for pass/code programs). Data only transformations had the lowest overhead at $1 \%$ size increase compared to original programs.

Figure 23 presents a summary of all metrics which include effectiveness and average overhead. Of note, a good majority of 
part 1 and 2 variants showed protections to be ineffective or defective. Techniques that were defective could mean that variants were better optimized for symbolic analysis. Data transformation in part 1 was the only technique alone to show an increase in analysis runtime on average, without any tests being defective, as seen in Figure 24. This suggests that Data obfuscation is a possible candidate for protection against symbolic execution tests. Part 2 tests did show some success for 3 character/digit protection, but it was not consistent and suggests the addition of Abstraction and Control Flow obfuscations decreased the effectiveness. The strongest test in part 2 was CAD, which was not as successful as Data obfuscation on its own.

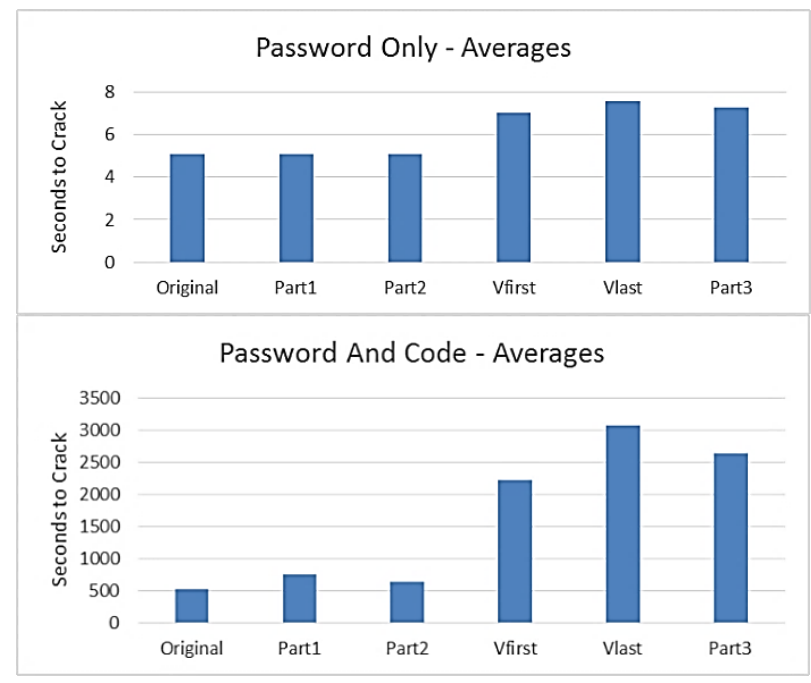

Figure 21. Password/code program average effectiveness

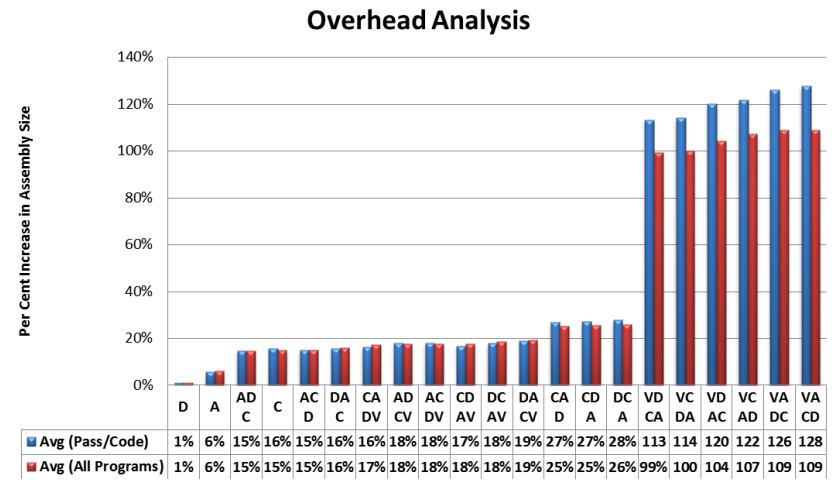

Figure 22. Average variant overhead based on number of assembly instructions

All tests in part 3 on average showed a significant increase in the obfuscation effectiveness. Virtualization on average was more successful if used last, for both password only and password/code protected programs. However, password/code protected variants where virtualization was used first all showed above a $200 \%$ increase in obfuscation effectiveness. In the case of 4 character/digit protected programs, they were more likely to be defective in virtualization-last variants than virtualization-first variants. For 4 character/digit protected programs, sequences of $\mathrm{ACDV}, \mathrm{ADCV}$, and DACV were all defective, while all virtualization-first transformations were effective. VACD, VADC, and CADV were the most successful on average, each average being above an hour to complete. VADC had the most success for 3 character/code protections where KLEE was set to expect 4 characters/digits, while VACD and CADV had the most success where KLEE was set to expect 6 characters/digits.

\begin{tabular}{|c|c|c|c|c|c|c|c|c|c|}
\hline & 6-char & 9-char & 12-char & 3-char $/ 2$ & 3-char $/ 3$ & 4-char/4 & $\begin{array}{c}\text { Effective } \\
\text { all }\end{array}$ & \begin{tabular}{|c|} 
Effective \\
pass\&code
\end{tabular} & $\begin{array}{c}\text { Average } \\
\text { Overhead }\end{array}$ \\
\hline A & $-1 \%$ & $0 \%$ & $0 \%$ & $83 \%$ & $-84 \%$ & $-33 \%$ & $x$ & $x$ & $6 \%$ \\
\hline C & $0 \%$ & $0 \%$ & $0 \%$ & $-75 \%$ & $-81 \%$ & $-54 \%$ & $x$ & $x$ & $1 \%$ \\
\hline D & $0 \%$ & $0 \%$ & $0 \%$ & $25 \%$ & $0 \%$ & $273 \%$ & $x$ & $x$ & $15 \%$ \\
\hline ACD & $0 \%$ & $0 \%$ & $0 \%$ & $92 \%$ & $-81 \%$ & $22 \%$ & $x$ & $x$ & $15 \%$ \\
\hline$A D C$ & $-1 \%$ & $0 \%$ & $0 \%$ & $92 \%$ & $-84 \%$ & $22 \%$ & $x$ & $x$ & $15 \%$ \\
\hline CAD & $-1 \%$ & $-1 \%$ & $0 \%$ & $-58 \%$ & $-77 \%$ & $178 \%$ & $x$ & $x$ & $25 \%$ \\
\hline CDA & $-1 \%$ & $-1 \%$ & $0 \%$ & $-8 \%$ & $-67 \%$ & $-42 \%$ & $x$ & $x$ & $25 \%$ \\
\hline DAC & $0 \%$ & $0 \%$ & $0 \%$ & $-33 \%$ & $-65 \%$ & $-20 \%$ & $x$ & $x$ & $16 \%$ \\
\hline DCA & $0 \%$ & $0 \%$ & $0 \%$ & $-8 \%$ & $-58 \%$ & $55 \%$ & $x$ & $x$ & $26 \%$ \\
\hline ACDV & $-1 \%$ & $-1 \%$ & $0 \%$ & $375 \%$ & $-28 \%$ & $271 \%$ & $x$ & $x$ & $18 \%$ \\
\hline ADCV & $-1 \%$ & $-1 \%$ & $0 \%$ & $366 \%$ & $-37 \%$ & $114 \%$ & $x$ & $x$ & $18 \%$ \\
\hline CADV & $97 \%$ & $98 \%$ & $0 \%$ & $250 \%$ & $51 \%$ & $898 \%$ & $x$ & $x$ & $17 \%$ \\
\hline CDAV & $195 \%$ & $98 \%$ & 99\% & $241 \%$ & $67 \%$ & $370 \%$ & $\checkmark$ & $\checkmark$ & $18 \%$ \\
\hline DACV & $0 \%$ & $-1 \%$ & $0 \%$ & $83 \%$ & $-9 \%$ & $71 \%$ & $x$ & $x$ & $18 \%$ \\
\hline DCAV & $98 \%$ & $0 \%$ & $0 \%$ & $208 \%$ & $81 \%$ & $543 \%$ & $x$ & $\checkmark$ & $19 \%$ \\
\hline VACD & $97 \%$ & $98 \%$ & $0 \%$ & $658 \%$ & $170 \%$ & $845 \%$ & $x$ & $\checkmark$ & $109 \%$ \\
\hline VADC & $97 \%$ & $98 \%$ & $0 \%$ & $1440 \%$ & $172 \%$ & $700 \%$ & $x$ & $\checkmark$ & $109 \%$ \\
\hline VCAD & $97 \%$ & $98 \%$ & $0 \%$ & $674 \%$ & $167 \%$ & $361 \%$ & $x$ & $\checkmark$ & $107 \%$ \\
\hline VCDA & $97 \%$ & $0 \%$ & $0 \%$ & $266 \%$ & $128 \%$ & $461 \%$ & $x$ & $\checkmark$ & $100 \%$ \\
\hline VDAC & $97 \%$ & $0 \%$ & $0 \%$ & $666 \%$ & $167 \%$ & $474 \%$ & $x$ & $\checkmark$ & $104 \%$ \\
\hline VDCA & $97 \%$ & $0 \%$ & $0 \%$ & $308 \%$ & $140 \%$ & $335 \%$ & $x$ & $\checkmark$ & $99 \%$ \\
\hline & \multicolumn{2}{|c|}{ Defective } & \multicolumn{2}{|c|}{ Ineffective } & \multicolumn{2}{|c|}{ Effective } & \multicolumn{2}{|l|}{ Effective } & \\
\hline & & $<-1 \%$ & $-1 t$ & to $1 \%$ & $>19$ & & $>500 \%$ & & \\
\hline
\end{tabular}

Figure 23. Effectiveness of transformation ordering

\section{Conclusions and Future Work}

In considering the data produced from our case study analysis and methodology, we can make a general observation that (as expected) dynamic transformation using virtualization provides higher obfuscation effectiveness when combined with other transformations, regardless of their ordering. When putting virtualization last in the ordering, we can conclude that effectiveness is possible to attain, but less consistent. In fact, the only potent transformation ordering in the study for all programs considered was CDAV, which on average only induced $18 \%$ overhead in variant size. When putting virtualization first, regardless of other category ordering, effectiveness is achievable on average, and in the case of our study, produced higher orders of magnitude in adversarial analysis time for symbolic analysis.

Another key takeaway from our study shows the potential impact of character size for passwords and activation codes, whether good or bad, on effectiveness. We would have assumed, for example, that a 12-character password program would have variants with more effectiveness, regardless of any ordering, but the data did not support this assumption. Effectiveness, however, was most prominent with large increases in analysis run-time when both a password and activation code are part of the original program, even for reasonable small sizes (2-4 digits for the code, for example). This may also allow us to conclude that original program composition is more of a key determinant for effectiveness than executive ordering. 
Inconsistencies in our results may be caused by KLEE's method for exploring branching paths in the executables. KLEE uses Random Path Selection to decide which path to explore, which could mean that increases in effectiveness could be related to the number of branches introduced into a program. [16] The random nature of branch selection could mean that repeating the same test could give different results. KLEE also uses the -optimization parameter in these tests, which runs LLVM optimization before testing the program. This was included to test the possibility that the obfuscations could be removed by optimization. The same optimizations were also used on the original programs, so it is uncertain if any deficiencies are a direct result of LLVM optimization.

In terms of confirming prior results from Banescu et al. in [26], they observe the highest KLEE analysis overhead for Tigress variants using transformations of Virt $\mathrm{x} 2$ (VV), AddO16-EncA (CD), EncA-Flat (DC), AddO16-Flat (CC), and combinations of flattening $(\mathrm{C}-)$. They also observe that flattening and virtualization increase effectiveness more than other transformation types. Our study shows that Control transformations alone are either ineffective or defective, but are lead to higher effectiveness when done before virtualization.

For future work, we plan to consider additional orderings (repeated applications of the same single category, double or repeated applications of transformations, etc.) and smaller permutations (AA, AC, $\mathrm{CA}, \mathrm{AD}, \mathrm{DA}$, etc.) to see what further effects are observable in executive ordering. We will also use longer password/code pairs, which require longer analysis times.

\section{Acknowledgments}

This material is based in part upon work supported by the National Science Foundation under Grants CNS-1305369 and DUE-1241675.

\section{References}

[1] D. McCandless. 2016. Million Lines of Code | Information is Beautiful. Informationisbeautiful.net. Available: http://www.informationisbeautiful.net/ visualizations/million-lines-of-code/

[2] A. Akhunzada, M. Sookhak, Nor B. Anuar, A. Gani, E. Ahmed, M. Shiraz, S. Furnell, A. Hayat, and M. K. Khan. 2015. Man-At-The-End Attacks. J. Netw. Comput. Appl. 48, C (February 2015), 44-57. DOI: 10.1016/j.jnca.2014.10.009

[3] C. Collberg and J. Nagra. 2009. Surreptitious Software: Obfuscation, Watermarking, and Tamperproofing for Software Protection (1st ed.). AddisonWesley Professional.

[4] B. Staggs. 2007. Implementing a Partial Serial Number Verification System in Delphi | Brandonstaggs.com. Available: http://www.brandonstaggs.com /2007/07/26/implementing-a-partial-serial-number-verification-system-indelphi/

[5] J. Cazalas, J. T. McDonald, T. R. Andel, and N. Stakhanova. 2014. Probing the Limits of Virtualized Software Protection. In Proceedings of the 4th Program Protection and Reverse Engineering Workshop (PPREW-4). ACM, New York, NY, USA, Article 5. DOI: 10.1145/2689702.2689707

[6] R. Rolles. 2009. Unpacking virtualization obfuscators. In Proceedings of the 3rd USENIX conference on Offensive technologies (WOOT'09). USENIX Association, Berkeley, CA, USA, 1-1.

[7] F. B. Cohen. 1993. Operating system protection through program evolution. Comput. Secur. 12, 6 (October 1993), 565-584. DOI: 10.1016/01674048(93)90054-9

[8] C. Collberg, C. Thomborson, and D. Low. 1997. A Taxonomy of Obfuscating Transformations. Technical Report 148, Department of Computer Science, University of Auckland.

[9] K. Heffner and C. Collberg. 2004. The obfuscation executive. In Information Security. Springer, 2004, pp. 428--440. DOI: 10.1007/978-3-540-30144-8_36

[10] C. Collberg, C. Thomborson, and D. Low. 1998. Manufacturing cheap, resilient, and stealthy opaque constructs. In Proceedings of the 25th ACM SIGPLAN-SIGACT symposium on Principles of programming languages (POPL '98). ACM, New York, NY, USA, 184-196. DOI:
$10.1145 / 268946.268962$

[11] Wu Zhou, Xinwen Zhang, and Xuxian Jiang. 2013. AppInk: watermarking android apps for repackaging deterrence. In Proceedings of the 8th ACM SIGSAC symposium on Information, computer and communications security (ASIA CCS '13). ACM, New York, NY, USA, 1-12. DOI: 10.1145/ 2484313.2484315

[12] radare. 2016. Radare.org. http://www.radare.org/r/

[13] GDB: The GNU Project Debugger. 2016. Gnu.org. Available: https://www.gnu.org/software/gdb/ .

[14] objdump - GNU Binary Utilities. 2016. Sourceware.org. Available: https://sourceware.org/binutils/docs/binutils/objdump.html.

[15] KLEE. 2016. Klee.github.io. Available: https://klee.github.io/

[16] C. Cadar, Daniel Dunbar, and Dawson Engler. 2008. KLEE: unassisted and automatic generation of high-coverage tests for complex systems programs. In Proceedings of the 8th USENIX conference on Operating systems design and implementation (OSDI'08), 209-224.

[17] LiME. 2016. Linux Memory Extractor. Available: https://github.com/ 504ensicsLabs/LiME

[18] B. Yadegari, B. Johannesmeyer, B. Whitely and S. Debray. 2015. A Generic Approach to Automatic Deobfuscation of Executable Code. In Proceedings of the IEEE Symposium on Security and Privacy, San Jose, CA, 2015, pp. 674691.

[19] M. Qu'eva. 2007. Phase-ordering in optimizing compilers. Technical University of Denmark.

[20] J. Macbride, C. Mascioli, S. Marks, Y. Tang, L. M. Head, and P. Ramach. 2005. A comparative study of java obfuscators. In Proceedings of the IASTED International Conference on Software Engineering and Applications (SEA 2005), 14-16.

[21] M. Ceccato, M. Penta, P. Falcarin, F. Ricca, M. Torchiano, and P. Tonella. 2014. A family of experiments to assess the effectiveness and efficiency of source code obfuscation techniques. Empirical Softw. Eng. 19, 4 (August 2014), 1040-1074.

[22] C. Collberg. 2016. The Tigress C Diversifier/Obfuscator Tigress.cs.arizona.edu. http://tigress.cs.arizona.edu/.

[23] Y. Kanzaki, A. Monden, and C. Collberg. 2015. Code artificiality: a metric for the code stealth based on an N-gram model. In Proceedings of the 1st International Workshop on Software Protection (SPRO '15). IEEE Press, Piscataway, NJ, USA, 31-37.

[24] S. Banescu, M. Ochoa, and A. Pretschner. 2015. A framework for measuring software obfuscation resilience against automated attacks. In Proceedings of the 1st International Workshop on Software Protection (SPRO '15). IEEE Press, Piscataway, NJ, USA, 45-51.

[25] A. Caliskan-Islam, R. Harang, A. Liu, A. Narayanan, C. Voss, F. Yamaguchi, and R. Greenstadt. 2015. De-anonymizing programmers via code stylometry. In Proceedings of the 24th USENIX Conference on Security Symposium (SEC'15), Jaeyeon Jung (Ed.). USENIX Association, Berkeley, CA, USA, 255-270.

[26] S. Banescu, C. Collberg, V. Ganesh, Z. Newsham, and A. Pretschner. 2016. Code obfuscation against symbolic execution attacks. In Proceedings of the 32nd Annual Computer Security Applications Conference (ACSAC 2016). ACM, New York, NY, USA, 189-200. doi: 10.1145/2991079.2991114

[27] Obfuscator LLVM. 2010. Available: https://github.com/obfuscator$\underline{\text { llvm/obfuscator/wiki }}$

[28] Y. Shoshitaishvili, R. Wang, C. Salls, N.Stephens, M. Polino, A. Dutcher, J. Grosen, S. Feng, C. Hauser, C. Kruegel, and G. Vigna. 2016. SoK: (State of) The Art of War: Offensive Techniques in Binary Analysis. In Proceedings of 2016 IEEE Symposium on Security and Privacy (SP), 138-157, IEEE. DOI: 10.1109/SP.2016.17 